\title{
Afrontamiento y sensibilidad emocional como moderadores de la relación estrés-salud en médicos venezolanos
}

\section{Coping and emotional sensitivity as moderators of the stress- health relationship in Venezuelan physicians}

\author{
Lisbeth Bethelmy ${ }^{1}$ \\ Escuela de Psicología, Universidad Católica Andrés Bello. Caracas -Venezuela \\ Leticia Guarino \\ Dpto. de Ciencia y Tecnología del Comportamiento, Universidad Simón Bolívar. \\ Caracas - Venezuela
}

(Recepción: Septiembre 2008 - Aceptación: Noviembre 2008)

\begin{abstract}
Resumen
La presente investigación tuvo como objetivo evaluar el rol moderador de la sensibilidad emocional y los estilos afrontamiento en la relación estrés-salud en médicos venezolanos. La muestra estuvo conformada por 130 médicos que trabajan en unidades críticas de hospitales públicos de la ciudad de Caracas, a quienes se evaluó su nivel de estrés laboral, sensibilidad emocional, estilos de afrontamiento y salud percibida. Las regresiones jerárquicas indicaron que los estilos de afrontamiento racional y por desapego emocional actúan como variables protectoras de los síntomas físicos, mientras que el afrontamiento por evitación constituye un factor de riesgo que acentúa los efectos negativos del estrés sobre la salud. El afrontamiento por evitación fue la única variable que actuó como moderadora de la relación entre el control percibido y los síntomas psicológicos. La sensibilidad emocional no mostró un efecto moderador, pero pudo establecerse su impacto directo sobre los síntomas. El estudio brinda nueva evidencia empírica para los modelos de sensibilidad emocional y afrontamiento para el estudio de las diferencias individuales en los profesionales de la medicina en Venezuela.

Palabras clave: afrontamiento, sensibilidad emocional, estrés laboral, médicos.
\end{abstract}

\begin{abstract}
The present research aimed at studying the moderator role of the emotional sensitivity and the coping styles in the stress-health relationship among Venezulean medical doctors. Sample was conformed by 130 physicians working in critical units of public hospitals in Caracas, who were assessed in their job stress, emotional sensitivity, coping styles and perceived health. Hierarchical regressions showed the rational and detached coping acting as protective moderators variables of the physical symptoms, while avoidance coping acts as a risk moderator factor increasing the pervasive effects of stress over health. Avoidance coping was the only variable which moderated the relationship between perceived control and psychological symptoms. Emotional sensitivity did not show a moderator effect, but rather a direct impact over symptoms could be established. This study brings new empirical evidence for the emotional sensitivity and coping model and for the study of the individual differences on health professionals in Venezuela.
\end{abstract}

Key words: coping, emotional sensitivity, work stress, physicians.

1 Correspondencia a: Msc. Lisbeth Bethelmy. Escuela de Psicología, Centro de Investigación y Formación Humanística, Universidad Católica Andrés Bello. Final Avenida Teherán, Montalbán, Caracas, Venezuela. E-mail: lbethelm@ucab.edu. ve 


\section{Introducción}

El estrés entendido como un tipo de emoción que involucra los factores psicológicos de la persona en interacción con las relaciones del entorno ha sido bien descrito por el Modelo Transaccional de Lazarus y sus colaboradores (1986; 1999; 2000), asumiendo que la evaluación e interpretación que hace el individuo de su entorno determina, en definitiva, lo que será o no estresante y la forma de lidiar con él.

En el ámbito laboral, el estrés ha sido asociado al autorreporte de deterioro en la salud física y psicológica (Lazarus, 1999). Entre los modelos revisados, el de estrés laboral de Karasek y Theorell (2000) ha mostrado suficiente evidencia empírica que apoya su poder predictivo a la hora de explicar los niveles de estrés laboral y su relación con la aparición de enfermedades, especialmente las de tipo coronario (Belzic, Landsbergis, Schnall \& Bake, 2004; Karasek, et al., 1988; Landsbergis, Schnall, Schwartz, Warren \& Pickering, 1995).

La combinación de valores altos y bajos de las demandas laborales y el control decisional producen cuatro escenarios diferentes del estrés laboral. La primera situación es denominada Tensión Laboral, en la cual las demandas psicológicas son altas y el control en la decisión es bajo, constituyéndose en la hipótesis principal del modelo; la segunda es llamada situación Activa, cuando la demanda laboral es alta y el control en la decisión también es alto; la tercera situación es la Baja Tensión, con una demanda psicológica baja y alto control en la decisión; y finalmente, la situación Pasiva, constituida por una baja demanda psicológica y bajo control decisional. Específicamente, se plantea una asociación significativa entre elementos o situaciones laborales que son psicológicamente demandantes e incontrolables y el incremento en la probabilidad del desarrollo subsecuente de enfermedades, principalmente coronarias. Así, una persona con altas demandas psicológicas en el trabajo y bajo control decisional en la tarea, tiene una mayor posibilidad de desencadenar reacciones psicológicas adversas para su estado de salud, tales como fatiga, ansiedad, depresión y enfermedades físicas en general (Karasek \& Theorell, 2000; O’Connor, O’Connor, White \& Bundred, 2000; Pelfrene, et al. 2001).

En este sentido, Karasek, et al. (1988) encontraron que la prevalencia de infartos al miocardio se diferenciaba de acuerdo a los tipos de trabajo u ocupación laboral y a los niveles de estrés laboral percibidos en cada uno. Específicamente, los profesionales de la medicina se encontraron en el cuadrante activo del modelo, mientras que aquellos de trabajo pesado, tales como técnicos, obreros, entre otros similares, clasificaron en el de estrés laboral, siendo este último grupo el de mayor prevalencia en la aparición de infartos al miocardio.

Sin embargo, en trabajos posteriores tanto Karasek y Theorell (2000) como O’Connor, et al., (2000), sugieren que en los últimos años las distribuciones de las ocupaciones laborales han ido cambiando como resultado de crisis económicas y sociales que modifican los niveles de demanda y control en algunas profesiones. Muy especialmente señalan a los profesionales de la medicina como un ejemplo de esa movilización al cuadrante de estrés laboral. Aunado a lo anterior, Chacón y Grau (2004); De las Cuevas (2003); Mingote, Moreno y Gálvez (2004); y Moreno, González y Garrosa (2001) también sugieren con tono alarmante la necesidad de establecer los niveles de salud y estrés en los profesionales de la medicina, debido a que se les considera actualmente como una población vulnerable a padecer enfermedades físicas y psicológicas originadas por el estrés laboral en el ejercicio de su profesión.

En este sentido, el trabajo del médico ha mostrado elementos más estresantes que otras profesiones, caracterizado por altas demandas laborales como por ejemplo enfrentar el sufrimiento de las personas y desarrollar su actividad muchas veces dentro de ambientes carentes de recursos que exigen una sobredemanda de atención a una gran diversidad de pacientes (Arnetz, 2001; Simpson \& Grant, 1991); situación que en algunas ocasiones se convierte en una praxis médica poco adecuada y poco asertiva (Firth-Cozens, 2001).

A pesar de la contundencia de estas evidencias, Karasek, et al. (1988) y Landsbergis, et al. (1995) fueron prudentes con los resultados de sus investigaciones y reconocieron la necesidad de 
estudiar otras variables que pudieran estar involucradas en la relación entre el estrés laboral, la ocupación y el estado de salud de los individuos; tales como variables de personalidad, variables genéticas o fisiológicas y variables socioambientales. En este sentido, Yayli, Yaman y Yaman (2003) y Dorz, Novara, Sica y Sanavio (2003) plantean una relación importante entre las estrategias de afrontamiento y el estrés crónico en los profesionales de la medicina, mientras que Mingote, et al. (2004) mostraron que la presencia de desgaste profesional o Burnout en médicos atiende a variables tales como: riesgos a manifestar enfermedad, tipo de especialización médica, cargo (residente o especialista), hábitos de vida, apoyo social (familiar y de trabajo), y muy especialmente, a la consideración de diferencias individuales involucradas, tales como los recursos de afrontamiento y la personalidad, que pueden impactar positiva o negativamente el estado de salud general.

En este sentido, son múltiples los autores que han dejado claro que es imposible comprender el fenómeno del estrés, sin considerar las variables moderadoras que vinculan a los estímulos o experiencias estresantes (inputs) con los resultados como adaptación, salud, enfermedad (ouputs) (ver p. ej. Baron \& Kenny, 1986; Guarino, 2005; Lazarus y Folman, 1986; Taylor \& Aspinwall, 1996). Para Cohen y Edwards (1989), la personalidad puede intervenir entre los eventos estresantes (o expectativas del evento) y la reacción del estrés, atenuando o acentuando la evaluación de éste como estímulo amenazador y, en conjunto con las estrategias de afrontamiento, actuar como factor moderador en el deterioro de la salud o mantenimiento del bienestar.

Una de las variables de personalidad que se ha venido estudiando recientemente en su rol moderador en la relación estrés-enfermedad es la Sensibilidad Emocional, propuesta por Guarino (2004; 2005; Guarino, Feldman y Roger, 2005; Guarino y Roger, 2005), encontrando que específicamente la dimensión de sensibilidad egocéntrica negativa se relaciona con reportes de deterioro en la salud autopercibida de estudiantes universitarios en períodos de adaptación (Guarino, 2004, 2005). Resultados posteriores apoyaron estas evidencias con cadetes (Herrera y Guarino, 2008) y con profesionales universitarios (Goncalves, Feldman y Guarino, 2008).

Con respecto a la interacción entre la sensibilidad emocional y el afrontamiento, Guarino (2004) sugiere que la sensibilidad interpersonal positiva ( constructo teóricamente relacionado con empatía), actuando en conjunto con el estilo de afrontamiento por desapego emocional debería funcionar como protectora de la salud, sobre todo en aquellas poblaciones laborales de servicio social de alta demanda, como es el caso de los profesionales de la salud, específicamente, los psicólogos clínicos, los médicos y las enfermeras, y que tal combinación debería ser una de las características esperadas en estas poblaciones.

Considerando lo anterior, se plantea como objetivo de la presente investigación determinar el posible efecto moderador de los estilos de afrontamiento y la sensibilidad emocional en la relación entre el estrés laboral y la salud autopercibida en médicos venezolanos, explorando así posibles patrones protectores o de riesgo al estrés en esta población laboral, la cual no había sido hasta ahora estudiada bajo los modelos teóricos descritos.

\section{Método}

\section{Participantes}

La muestra estuvo constituida por 130 médicos, de diferentes categorías laborales: residentes (70,8\%), adjuntos (22,3\%) y jefes de servicio (6,9\%). De éstos, 75 fueron mujeres y 55 hombres, distribuidos en las unidades de medicina interna, nefrología, cirugía general, cardiología, oncología y terapia intensiva, pertenecientes a cuatro hospitales del área metropolitana de la ciudad de Caracas. Las edades estuvieron comprendidas entre los 25 y los 63 años, con una media de 35 años $(\mathrm{Dt}=9.3)$. 


\section{Instrumentos}

- Lista de Chequeo del Estado de Salud (Health Status Checklist), versión adaptada y validada por Guarino (2004; 2005). Permite medir la salud física y psicológica autopercibida de los participantes, quienes deben indicar los cambios que han experimentado en el último mes con respecto a una lista predeterminada de síntomas, señalando si su estado de salud se ha mantenido, empeorado o mejorado con respecto a los últimos 6 meses, según la siguiente escala: “Completamente mejor”, “mucho mejor”, “un poco mejor”, “un poco peor”, “mucho peor", "completamente peor", "no aplica”. El instrumento comprende 2 dimensiones: a) Síntomas psicológicos y dolencias crónicas (10 ítems, $\alpha=0.81, \mathrm{~N}=419)$; y b) Síntomas físicos y dolencias agudas ( 7 ítems, $\alpha=0.68, \mathrm{~N}=419$ ) (ver Guarino, 2005). Igualmente es importante destacar que existen 2 ítems que solo son aplicables a mujeres, por lo que hay diferencias en la corrección e interpretación de este inventario para ambos sexos.

- Escala de Sensibilidad Emocional -ESE- (Guarino y Roger, 2005), esta escala mide la responsividad o reactividad emocional de los individuos a través de 45 ítems agrupados en tres dimensiones, a saber: Sensibilidad Egocéntrica Negativa -SEN- (22 ítems), Distanciamiento Emocional - DE- (10 ítems) y Sensibilidad Interpersonal Positiva-SIP- (13 ítems). Los autores reportan índices test-retest para las tres dimensiones de entre $r^{t t}=0,65 \mathrm{y} r^{t t}=0,72$, mientras que las consistencias internas se ubican en $\alpha=0,824$ para SEN; $\alpha=0,742$ para DE y $\alpha=0,71$ para SIP, para una muestra de $N=419$ sujetos, respectivamente.

- Cuestionario de Afrontamiento (Coping Style Questionnarie- CSQ, Roger, Jarvis \& Najarian, 1993), versión adaptada y validada a la población venezolana por Guarino, Sojo y Bethelmy (2007). Este cuestionario mide cuatro diferentes dimensiones de afrontamiento: emocional - EMO (11 ítems), racional - RAC (11 ítems), evitación- EVI (5 ítems) y desapego emocional DES (13 ítems). El instrumento mostró índices de consistencia interna bastante aceptables (EMO $\alpha=0.78$; RAC $\alpha=0.76$; EVI $\alpha=0.65$; y DES $\alpha=0.73$ para $\mathrm{N}=292$, respectivamente).

- Cuestionario de Estrés Laboral de Karasek y Theorell, traducido al español y adaptado por Pérez (1997) de la versión original del año 1990. El cuestionario mide la percepción personal del ambiente laboral, cantidad de trabajo realizado, satisfacción con el trabajo, apoyo del supervisor y compañeros. Para la presente investigación sólo se analizaron las subescalas de Demandas Laborales $(\alpha=0,55)$, y Control Decisional $(\alpha=0,73)$, componentes del estrés laboral por definición.

\section{Procedimiento}

Se solicitó inicialmente el permiso respectivo en cada una de las unidades de servicio de los hospitales estudiados, a través de visitas y entrevistas a los jefes de servicio de cada unidad, así como a algunos de los subdirectores de los hospitales. Aceptada la petición de llevar a cabo la investigación en la unidad, se pautaron fechas para la aplicación de los instrumentos a la mayor cantidad de médicos posibles por sesión.

\section{Resultados}

Análisis descriptivo de las variables psicosociales y de salud

Los resultados de la Tabla 1 nuestra que los médicos reportan bajos niveles de síntomas físicos y psicológicos, considerando el rango de valores obtenidos y posibles (0-31; 0-42, respectivamente). Resultados similares se presentan con respecto a los síntomas psicológicos, tanto para el grupo total como por géneros; asumiéndose un estado de salud de regular a bueno para todos los participantes del estudio.

Por su parte, se observan bajos puntajes de sensibilidad egocéntrica negativa, valores intermedios de distanciamiento emocional y altos valores de sensibilidad interpersonal positiva. Con respecto 
al afrontamiento, los médicos utilizan el afrontamiento emocional de manera regular, con una alta frecuencia el afrontamiento racional, medianamente el afrontamiento por desapego emocional y una tendencia creciente a utilizar el afrontamiento por evitación.

Además, los médicos perciben estar sometidos a altas demandas laborales pero con un alto control, sugiriendo así la percepción de adecuado control e independencia en sus actividades laborales, al menos en los aspectos constitutivos referidos a las destrezas laborales y la toma de decisiones. Sólo 19 médicos resultaron bajo la condición extrema de estrés, aplicando la fórmula propuesta por Karasek y Theorell (2000), con la cual se seleccionan sólo aquellos puntajes por encima de la mediana de demandas laborales y por debajo de la mediana de control.

Tabla 1. Estadísticos Descriptivos de las Variables del Estudio

\begin{tabular}{|c|c|c|c|c|c|c|}
\hline & Media & Dt & As & $\mathbf{K}$ & $\begin{array}{c}\text { Rango } \\
\text { obtenido }\end{array}$ & $\begin{array}{l}\text { Rango } \\
\text { posible }\end{array}$ \\
\hline $\begin{array}{c}\text { Síntomas Psicológicos } \\
\text { mujeres }\end{array}$ & 16,5 & 13,36 & 0,83 & 0,11 & $0-51$ & $0-60$ \\
\hline $\begin{array}{c}\text { Síntomas Psicológicos } \\
\text { hombres }\end{array}$ & 10,75 & 10,13 & 1,15 & 1,12 & $0-42$ & $0-54$ \\
\hline $\begin{array}{l}\text { Síntomas físicos } \\
\text { Síntomas Psicológicos }\end{array}$ & 5,41 & 6,99 & 1,55 & 1,97 & $0-31$ & $0-42$ \\
\hline Total & 13,6 & 11,65 & & 0,164 & $0-47$ & $0-54$ \\
\hline $\begin{array}{c}\text { Sensibilidad } \\
\text { Egocéntrica Negativa }\end{array}$ & 6,96 & 4,8 & 0,64 & $-0,48$ & $0-20$ & $0-22$ \\
\hline $\begin{array}{l}\text { Distanciamiento } \\
\text { Emocional }\end{array}$ & 4,04 & 2,92 & 0,178 & - 0,72 & $0-10$ & $0-10$ \\
\hline $\begin{array}{c}\text { Sensibilidad } \\
\text { Interpersonal Positiva }\end{array}$ & 7,59 & 2,55 & $-0,541$ & $-0,109$ & $1-13$ & $0-13$ \\
\hline Afrontamiento & & & & & & \\
\hline Emocional & 21,9 & 3,86 & 0,95 & 1,76 & $13-36$ & $12-48$ \\
\hline Afrontamiento Racional & 30,7 & 4,42 & 0,042 & $-0,48$ & $20-40$ & $10-40$ \\
\hline Afrontamiento & & & & & & \\
\hline $\begin{array}{l}\text { por Desapego } \\
\text { emocional }\end{array}$ & 28,9 & 5,5 & 0,703 & 1,15 & $18-51$ & $12-52$ \\
\hline $\begin{array}{l}\text { Afrontamiento de } \\
\text { Evitación }\end{array}$ & 11,8 & 3,1 & 0,15 & $-0,38$ & $5-20$ & $5-20$ \\
\hline Demanda Laboral & 34,3 & 5 &, 27 & 0,57 & $18-48$ & $12-48$ \\
\hline Control & 38,3 & 4,87 & $-0,82$ & 0,37 & $23-45$ & $15-45$ \\
\hline
\end{tabular}




\section{Regresión Jerárquica para el análisis de la moderación}

Una vez determinado el cumplimiento de los supuestos para realizar el análisis de regresión, se calcularon las ecuaciones para la variable salud en sus dos factores, síntomas físicos y síntomas psicológicos.

Con respecto a la relación entre las demandas laborales y los síntomas físicos, los resultados indicaron que los factores psicosociales evaluados no tuvieron un rol moderador en dicha relación. Sin embargo, la sensibilidad egocéntrica negativa, el afrontamiento emocional y el afrontamiento por evitación tuvieron un efecto directo positivo, a la vez que el afrontamiento racional obtuvo un efecto directo negativo en la explicación del reporte de los síntomas físicos. Específicamente, la sensibilidad egocéntrica negativa obtuvo una varianza explicada total del $2 \%$, indicando una correlación baja y positiva entre sensibilidad egocéntrica y síntomas físicos $(\beta=0,256$; $p=0,003)$. El afrontamiento emocional y el de evitación también muestran una correlación baja y significativa ( $\beta=0,267 ; p=0,002$ y $\beta=0,181 ; p=0,039$, respectivamente) y con una varianza explicada total de $9,2 \%$ y $5,5 \%$, respectivamente, de forma tal que a mayores niveles de afrontamiento emocional y de evitación, mayores los reportes de síntomas físicos.

En la evaluación de la relación entre el control y los síntomas físicos, las variables afrontamiento racional, por desapego emocional y afrontamiento por evitación sí mostraron un efecto moderador. La interacción entre afrontamiento racional y control resultó significativa $(\beta=-2,75 ; p=0,017)$, explicando el $8,9 \%$ de la varianza total para síntomas físicos; además predice directamente la aparición de estos síntomas con un 4,6\% de la varianza total explicada, mostrando que a niveles elevados de afrontamiento racional, menores los niveles de síntomas de salud reportados $(\beta=-0,209$; $\mathrm{p}=0,019$ ). En la figura 1 puede visualizarse el efecto de interacción entre el afrontamiento racional y el control sobre los síntomas físicos, lo cual sugiere el rol protector de este tipo de afrontamiento en conjunto con una percepción de control sobre la salud física de los médicos.

Figura 1. Interacción de Control x Afrontamiento Racional en la moderación de Sintomas Físicos

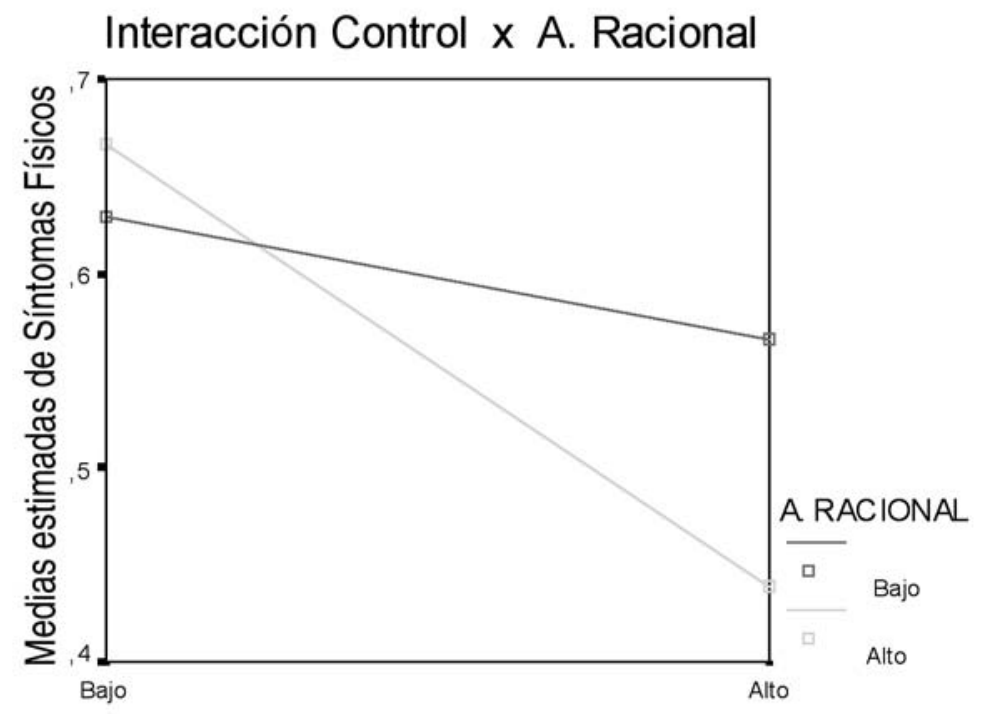

CONTROL 
El segundo efecto moderador lo muestra el afrontamiento por desapego emocional en la relación entre control y síntomas físicos (cambio en $\mathrm{R}^{2}=0,033$; sig=0,039), explicando el 4,3\% de la varianza ( $\beta=-1,874 ; p=0,039)$. La interacción puede visualizarse en figura 2, los síntomas físicos disminuyen considerablemente cuando, tanto el control como el afrontamiento por desapego son altos, mostrándose este estilo de afrontamiento como un factor protector de la salud física autopercibida.

Figura 2. Interacción de Control x Afrontamiento por desapego en la moderación de los Síntomas Físicos.

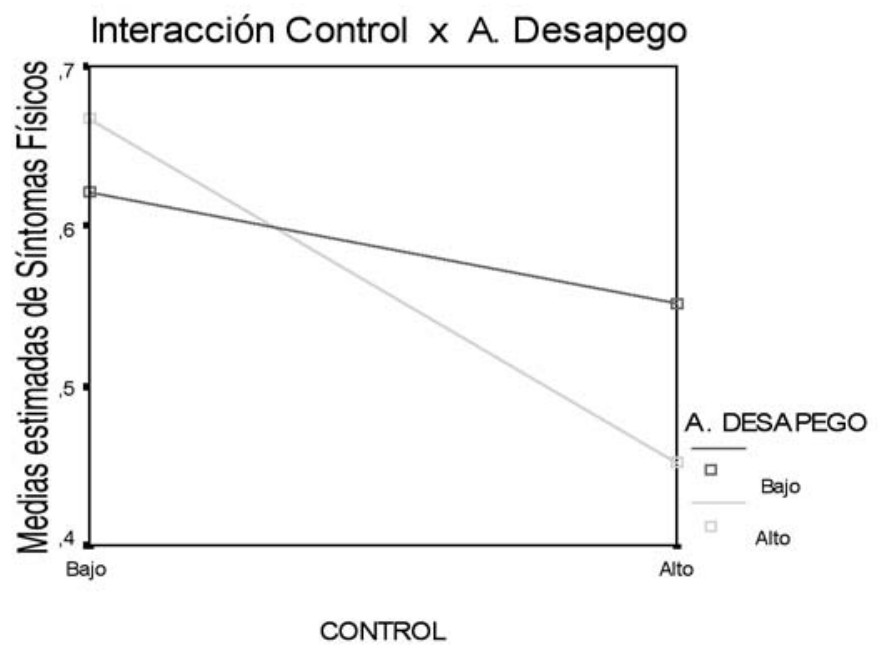

A su vez, el afrontamiento por evitación actúa como una variable moderadora entre el control y la salud física (cambio $\mathrm{R}^{2}=0,034$; sig=0,034), explicando significativamente el 6,5\% de la varianza total de la interacción de las variables para predecir síntomas físicos $(\beta=-1,69 ; \mathrm{p}=0,034)$. El efecto de interacción se observa en la figura 3, así, independientemente de los valores de control, el uso del afrontamiento por evitación genera altos reportes de síntomas físicos; alcanzando dichos síntomas su más alto nivel cuando confluyen los reportes de bajo control y frecuente uso de afrontamiento por evitación.

Figura 3. Interacción de Control x Afrontamiento por Evitación en la moderación de los Síntomas Físicos

Interacción Control X A. Evitación

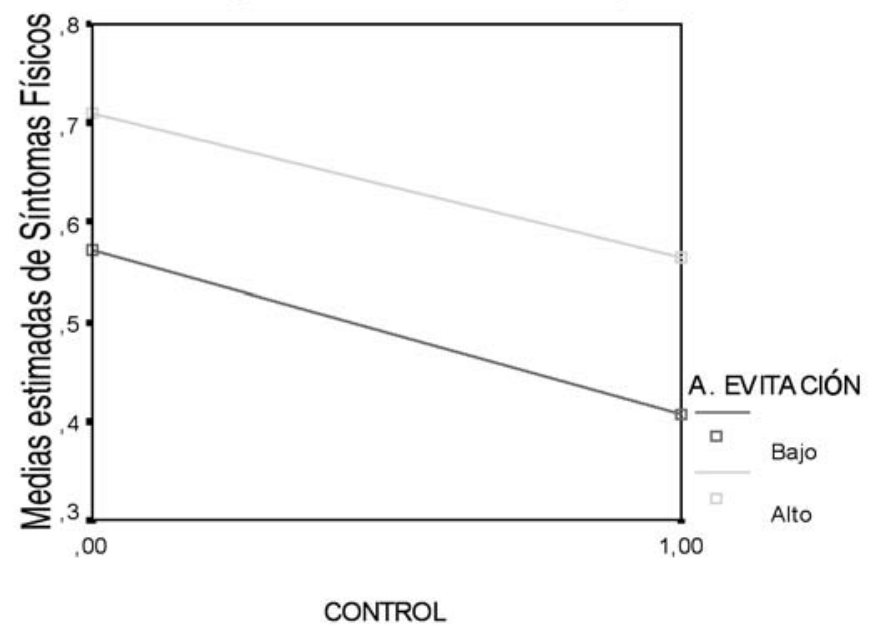


Para los análisis del efecto moderador de los síntomas psicológicos se introdujo la variable sexo, con el objetivo de evaluar si existen diferencias de género en cuanto a la manifestación de estos síntomas y su interacción con el estrés laboral y los factores psicosociales. Los resultados indican que solamente el afrontamiento por evitación ejerce un rol moderador en dicha relación, explicando significativamente el 14,4\% de la varianza total (ver tabla 2). También se puede observar que tanto el sexo como el control ejercieron un efecto directo significativo en la explicación de los síntomas psicológicos, en el sentido de que percibir un escaso control decisional y ser mujer, explican significativamente la aparición de síntomas físicos, con una varianza total de 10,6\% y $4,6 \%$, respectivamente.

Tabla 2. Análisis de Regresión Jerárquica entre Control, Afrontamiento por Evitación y Síntomas Psicológicos.

\begin{tabular}{|c|c|c|c|c|c|c|c|c|}
\hline \multirow[b]{2}{*}{ Modelo } & & \multicolumn{7}{|c|}{ Síntomas Físicos } \\
\hline & & $\bar{\beta}$ & $\bar{T}$ & $\bar{p}$ & $\mathrm{R}^{2}$ & $\begin{array}{l}\text { Cambio } \\
\text { en } R^{2}\end{array}$ & $\begin{array}{l}\text { Cambio } \\
\text { en F }\end{array}$ & Sig \\
\hline \multirow{2}{*}{$\begin{array}{l}1 \\
2\end{array}$} & Sexo & 0,213 & 2,470 & 0,015 & 0,046 & 0,046 & 6,102 &, $015^{*}$ \\
\hline & $\begin{array}{l}\text { Control } \\
\text { Afrontamiento } \\
\text { por evitación }\end{array}$ & $\begin{array}{r}-0,061 \\
0,219\end{array}$ & $\begin{array}{c}-0,70 \\
2,597\end{array}$ & $\begin{array}{l}0,489 \\
0,011\end{array}$ & 0,106 & 0,061 & 4,282 & $0,016^{*}$ \\
\hline \multirow[t]{4}{*}{3} & Sexo & 0,203 & 2,453 & 0,016 & & & & \\
\hline & Cont1 & 0,647 & 1,915 & 0,058 & & & & \\
\hline & $\begin{array}{l}\text { Atrontamiento } \\
\text { por evitación }\end{array}$ & & & & & & & \\
\hline & $\begin{array}{l}\text { Control } \mathrm{X} \\
\text { Afrontamiento } \\
\text { por evitación }\end{array}$ & $-1,773$ & $-2,33$ & 0,021 & 0,144 & 0,037 & 5,450 & $0,021^{*}$ \\
\hline
\end{tabular}

*significativo a $\mathrm{p}<0,05$

La figura 4 muestra la interacción significativa entre el control y el afrontamiento por evitación en relación con los síntomas psicológicos para todos los integrantes de la muestra, observándose que independientemente de los niveles de control, cuando el afrontamiento por evitación es alto se obtienen altos niveles de síntomas psicológicos.

Figura 4. Interacción Control x Afrontamiento por Evitación en la moderación de los Síntomas Psicológicos

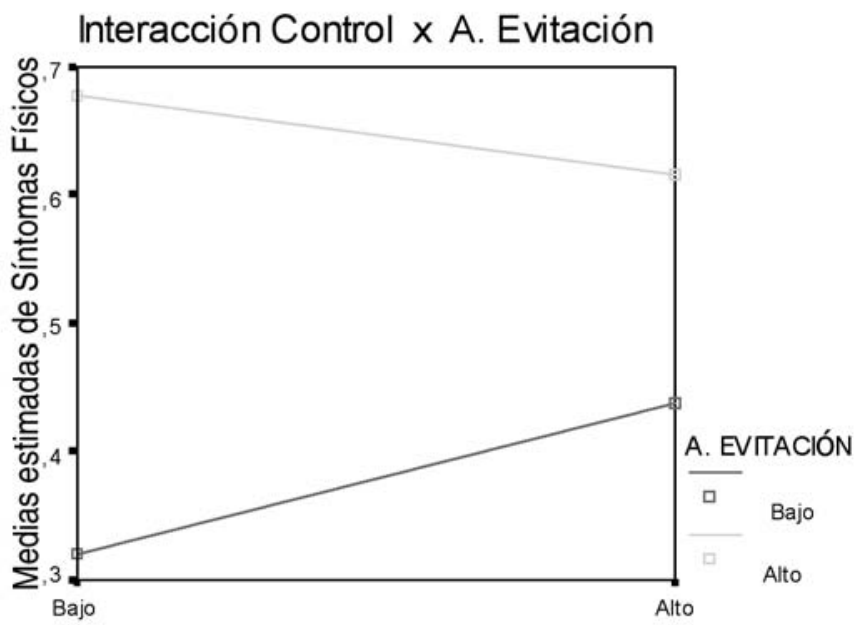

CONTROL 
Por último, las variables sensibilidad egocéntrica negativa, demandas laborales y estilos de afrontamiento explicaron de manera directa la aparición de síntomas psicológicos. Específicamente, la sensibilidad egocéntrica negativa $(\beta=0,418 ; \mathrm{t}=5,4 ; \mathrm{p}=0,000)$, el afrontamiento emocional $(\beta=0,505 ; t=6,8 ; p=0,000)$ y el afrontamiento por evitación $(\beta=0,242 ; t=2,9 ; p=0,004)$ lo hicieron de manera positiva, mientras que el afrontamiento racional $(\beta=-0,179 ; \mathrm{t}=-2,11 ; \mathrm{p}=0,037)$, por desapego emocional $(\beta=-0,183 ; t=-2,19 ; p=0,030)$ y las demandas laborales $(\beta=-0,158 ; t=-2,051 ; p=0,042)$ lo hicieron de manera negativa. La sensibilidad interpersonal positiva y el distanciamiento emocional (dimensiones de la sensibilidad emocional) no aportaron en este estudio ninguna evidencia de moderación o efecto directo sobre la salud de los médicos.

\section{Discusión}

El objetivo principal de la presente investigación fue determinar si las dimensiones de sensibilidad emocional y las de afrontamiento actuaban como moderadoras de la relación entre el estrés laboral y la salud física y psicológica de los médicos(as), basado en el modelo transaccional del estrés según el cual variables de índole personal podrían actuar como moderadoras de tal relación.

El principal hallazgo fue encontrar que los estilos de afrontamiento racional, por desapego emocional y por evitación actuaron como variables moderadoras de la relación entre el control decisional (componente del estrés laboral) y los síntomas físicos autopercibidos, evidenciándose además que sólo el afrontamiento por evitación actuó como moderador de la relación entre el control y los síntomas psicológicos de los médicos y médicas. Así, los estilos de afrontamiento, además de ejercer un efecto directo sobre la salud, ejercen un efecto interactivo en la relación, es decir, su presencia exacerba o disminuye el efecto del control laboral percibido sobre la salud reportada por los médicos. Específicamente, el uso del afrontamiento racional y por desapego emocional amortiguan el efecto perjudicial que el bajo control laboral tiene sobre la salud física autopercibida, mientras que el uso del afrontamiento por evitación perjudica el estado de salud, tanto física como psicológica, sobre todo en las circunstancias en las que el médico percibe que tiene un bajo control sobre su medio laboral.

Este resultado brinda un soporte empírico para el modelo de control emocional de Roger (1995), en el que los estilos de afrontamiento y otras diferencias individuales pueden ejercer un rol moderador en la relación entre el estrés y la salud autopercibida, aunque en este estudio no se comprobó este mismo efecto para la sensibilidad emocional.

El hecho de que las estrategias de afrontamiento hayan resultado moderadoras en la explicación de la naturaleza de la relación estrés-salud y no las variables de personalidad (en este estudio la sensibilidad emocional), apoya la hipótesis de Steptoe (1991a, 1991b), quien defiende que es el afrontamiento el que está más relacionado con las situaciones de estrés que la misma personalidad, debido a dos motivos fundamentales: a) las variables de afrontamiento están determinadas por varios factores relacionados con el contexto social y cultural en el que se desarrolla el evento estresante (en este caso el contexto responde a los hospitales y al entorno y actividades que los caracteriza) y b) personas con diferentes personalidades pueden utilizar en determinados momentos las mismas estrategias de afrontamiento y si además, éstas resultan adaptativas hasta pudieran convertirse en un hábito.

De igual forma, Guarino, et al. (2007) sostienen que los estilos de afrontamiento entendidos bajo el modelo de Control Emocional de Roger y evaluados según el instrumento ideado por este autor, se comportan en forma más global y estable para hacer frente a las características del estresor, a diferencia de su caracterización contextual como se había estado entendiendo al afrontamiento habitualmente. En este sentido, Gaher, Simona, Jacobs, Meyer y Jonhson (2005) encontraron una cierta relación de moderación entre la afectividad negativa y los estilos de afrontamiento; sin embargo, en el reporte de Gaher, et al. (2005) fue el modelo mediador el que permitió explicar la intervención de la afectividad negativa; dejando abierta la posibilidad de la acción de los dos modelos -mediación y moderación-. Bolger (1990) también planteó una relación de moderación del afrontamiento en 
la relación entre personalidad y salud, encontrando que los estilos adaptativos de afrontamiento actúan como moderadores entre el neuroticismo y la ansiedad. En síntesis, características como la sensibilidad emocional pudieran no tener un impacto directo en la relación estrés-salud (moderación), sino más bien indirecto (mediación), influyendo sobre las formas de afrontamiento que adoptan los individuos. Esta es sin duda una posibilidad que debe seguirse explorando.

Sin embargo, las evidencias de la relación entre la sensibilidad emocional y el deterioro de salud de los médicos fueron consistentes con lo reportado por Cohen y Edwards (1989), quienes plantearon que algunas características de personalidad como el neuroticismo ejercen un rol directo sobre los reportes de salud de las personas. En este sentido, aún no habiéndose apoyado con los resultados presentes la hipótesis de moderación de la sensibilidad emocional, esta característica sí permite explicar un aumento en el reporte de síntomas físicos y psicológicos en los médicos, tal como ya se ha observado en otros trabajos recientes (Goncalves et al., 2008; Herrera y Guarino, 2008 ), brindando así un soporte empírico a la propuesta de Guarino (2004, 2005), quien sugiere que específicamente la sensibilidad egocéntrica negativa constituye una característica personal nociva para el estado de salud general de los individuos, en la medida en que los predispone a una mayor reactividad fisiológica frente a los estresores, con su consecuente impacto sobre el bienestar.

Adicionalmente, se observó que el efecto protector de la salud de los médicos no viene dado por poseer características empáticas, como reportan Austin, Evans, Goldwater y Potter (2005) o por la manifestación de sensibilidad interpersonal positiva, sino por la utilización en conjunto de estrategias de afrontamiento por desapego emocional y racional, y el uso reducido de la estrategia de evitación en situaciones calificadas como estresantes o de poco control laboral. Específicamente, la percepción de un alto control y la utilización del afrontamiento racional y por desapego emocional protegen a los individuos de desarrollar síntomas de deterioro físico. En este sentido, Guarino (2004; 2005) señala que esta manifestación de la empatía, aunque deseable en los profesionales de la medicina, no siempre es positiva si no va acompañada de estrategias de afrontamiento adecuadas, especialmente las de desapego emocional, las cuales minimizan los efectos negativos del involucramiento personal en las emociones de los otros.

La adopción de la estrategia de afrontamiento racional por todos los médicos multiplica el efecto protector del alto control sobre la salud. Tal como lo establecieron Endler y Parker (1991), Lazarus y Folkman (1986) y Roger, et al. (1993), la adopción de la estrategia racional consiste en la búsqueda de todas las alternativas posibles para la solución real de las desavenencias generadas en un entorno estresante. Para este caso en particular, los médicos están en la búsqueda constante y dinámica de soluciones alternativas al bajo control decisional que pudieran estar percibiendo en su ambiente laboral y personal, aminorando además, el impacto negativo de este bajo control sobre la salud.

De igual manera, el afrontamiento por desapego fue concebido por Roger, et al. (1993), como la estrategia que separa de una manera adaptativa las emociones propias de la de los otros, no dejando que las emociones y situaciones ajenas perturben de manera negativa el equilibrio interno personal y emocional. Así, los médicos que adoptan esta estrategia de afrontamiento son capaces de no involucrarse emocionalmente en situaciones en las cuales no es posible obtener el control en la toma de decisiones y en las acciones concernientes a las destrezas laborales exigidas. Igualmente, es una estrategia que en conjunto con la percepción de un alto control laboral potencia el efecto protector sobre la salud de los médicos. Mismos resultados encontraron Dorz, et al. (2003), también en el ámbito hospitalario, mostrando que el uso de estrategias adaptativas, como el sentido del humor, predicen bajos niveles de depresión en médicos oncólogos.

Un patrón de riesgo para la salud, tanto física como psicológica, lo constituye la utilización del afrontamiento por evitación, independientemente de la percepción de los médicos de sus niveles de control decisional. Roger, et al., (1993), propusieron al afrontamiento por evitación como uno de los factores de alto riesgo para preservar un buen estado de salud frente a las experiencias estresantes, ya que este tipo de afrontamiento implica que los individuos se distancien de sus problemas reales, sin resolverlos, dejando de tomar las debidas acciones para la disminución de las consecuencias negativas que éstos acarrean. En el caso del bajo control, es muy probable que los médicos que 
adopten este tipo de afrontamiento no movilicen sus esfuerzos para resolver las situaciones en las que vean amenazados el poder de decisión y la percepción de minimización de las propias destrezas laborales, situación que se agrava en las mujeres. Para Matud (2004), las mujeres son las que utilizan en mayor medida el estilo de afrontamiento por evitación; sin embargo Rout (1999) reportó que este estilo es mayormente adoptado por los hombres; encontrándose así diferencias de género en cuanto a la vulnerabilidad al deterioro de la salud relacionado con la acción de evitar el suceso aversivo.

La presente investigación brinda apoyo empírico para el rol moderador solamente de los estilos de afrontamiento, no así para la sensibilidad emocional en la relación entre el estrés laboral y la salud, como había sido propuesto por algunos autores (Cohen \&Edwards, 1989; Guarino, 2005; Matthews \& Deary, 1998; Smith \& Williams, 1992). Sin embargo, en función de los resultados actuales, pudiera suponerse que la sensibilidad emocional, como variable de personalidad, parece tener más bien un efecto de mediación en la relación entre el estrés y la salud y no un efecto de moderación, como hasta ahora se había descrito en el modelo (ver Guarino 2004; 2005), por lo que deberá probarse tal efecto en futuras investigaciones. Una hipótesis factible es que la sensibilidad emocional actúa determinando la evaluación cognitiva que hacen los individuos ante los estímulos y sobre los recursos de afrontamiento que despliegan, lo cual hablaría más bien de un efecto indirecto y de mediación. En el presente se están desarrollando estudios precisamente para comprobar esta nueva hipótesis.

La relevancia del presente estudio se basa en el aporte de nuevas evidencias acerca del comportamiento y las implicaciones de los componentes del estrés sobre la salud autopercibida de una muestra de médicos y médicas venezolanos(as), así como también en el soporte empírico y teórico de los modelos que proponen a las variables de personalidad y de afrontamiento al estrés como constructos subyacentes de la vivencia asociada al bienestar físico y psicológico de las personas.

\section{Referencias}

Arnetz, B. (2001). Psychological Challenges Facing Physicians of Today. Social Science and Medicine, 52, 203-213.

Austin, E., Evans, P., Golgwater, R, \& Potter, V. (2005). A Preliminary Study of Emotional Intelligence, Empathy and Exam Performance in First Year Medical Students. Personality and Individual Differences, 39, 1395-1405.

Baron, R.M. \& Kenny, D.A. (1986). The moderator mediator variable distinction in social psychological research-conceptual, strategic, and statistical considerations. Journal of Personality and Social Psychology, 51, 1173-1182.

Belzic, K., Landsbergis, P., Schnall, P. \& Baker, D. (2004). Is Job Strain a Mayor of Cardiovascular Disease Risk? Scandinavian Journal Work Environmental Health, 30 (2), 85-128.

Bolger, N. (1990). Coping as a Personality Process: A Prospective Study. Journal of Personality and Social Psychology, 59 (3), 525-537.

Chacón, M. y Grau, J. (2004). Burnout y variables personales moduladoras en enfermeros que trabajan en hospitales oncológicos. Psicología y Salud. 14 (1), 67-78.

Cohen, S. \& Edwards, J. (1989). Personality Caracteristics as moderators of the relationship between stress and disorder. En R. Neufeld (Ed). Advances in the investigation of psychological stress. New York: John Wiley and Sons Ltd.

De las Cuevas, C. (2003). Burnout en los profesionales de la atención a las drogodependencias. Trastornos Adictivos, 5 (2): 107-113.

Dorz, S., Novara, C., Sica, C. \& Sanavio, E. (2003). Predicting Burnout among HIV/AIDS and Oncology Health Care Workers. Psychology and Health. 18, 677-684. 
Endler, N. \& Parker, J. (1990). Multidimensional Assessment Of Coping: A Critical Evaluation. Journal of Personality of Social Psychology. 58, 844-854.

Firth-Cozens, J. (2001). Interventions to improve physicians' well-being and patient care. Social Science and Medicine, 52, 215-222.

Gaher, R. Simons, J, Jacobs, G, Myer, D. \& Jonhson-Jiménez, E. (2006). Coping motives and trait negative affect: Testing mediation and moderation models of alcohol problems among American Red Cross disaster workers who responded to the September 11, 2001 terrorist attacks. Addictive Behaviors, 31, 1319-1330.

Goncalves, L., Feldman, L. y Guarino, L. (2008, Julio). Job strain, Negative Sensitivity and health in Venezuelan professionals. Comunicación presentada en la XXIX International Congress of Psychology, Berlín, Alemania.

Guarino, L. (2004). Emocional Sensitivity: a new measure of emotional lability and its moderating role in the stress-illness relationship. Tesis doctoral sin publicar, Universidad de York, Inglaterra.

Guarino, L. (2005). Sensibilidad Emocional: Su Rol Moderador en la Relación Estrés-Enfermedad. Psicología Conductual, 13 (2), 217-230.

Guarino, L. y Roger, D. (2005). Construcción y Validación de la Escala de Sensibilidad Emocional (ESE). Un nuevo enfoque para medir neuroticismo. Psicothema, 17 (3), 465-470.

Guarino, L., Feldman, L. y Roger D. (2005). La diferencia de la sensibilidad emocional entre británicos y venezolanos. Psicothema, 17, 633-638.

Guarino, L., Sojo, V. y Bethelmy, L. (2007). Adaptación y validación preliminar de la versión hispana del CSQ (Coping Style Questionnaire). Psicología Conductual, 15, 173-189.

Herrera, V. y Guarino, L. (2008). Personalidad, estrés y salud en cadetes navales venezolanos. Universitas Psicológica, 7, 1, 185-198.

Karasek, R. \& Theorell, T. (2000). The Demand-Control-Support Model and CVD. En L. Lennart (Ed.) Stressor al the workplace: Theoretical Models. Occupational Medicine. 15 (1). 69-103.

Karasek, R., Theorell, T., Schwartz, J., Schnall, P., Pieper, C. \& Michela, J. (1988). Job Characteristics in Relation to the Prevalence of Myocardial Infarction en the US Health Examination Survey (HES) and the Health and Nutrition Examination Survey (HANES). American Journal of Public Health. 78 (8), 910-918.

Landsbergis, P., Schnall, P., Schwartz, J., Warren, K. \& Pickering, T. (1995). Job Strain, Hypertension, and Cardiovascular Disease: Empirical Evidence, Methodological Issues, and Recommendations for Future Research. En S. Sauter y L. Murphy (Eds.) Organizational Risk Factors for Job Stress. Washington: APA

Lazarus, R. (1999). Stress and Emotion. A new synthesis. London: Free Association Books.

Lazarus, R. (2000). Estrés y Emoción, manejo e implicaciones para la salud. Bilbao: Desclèe de Brouwer.

Lazarus, R. y Folkman, S. (1986). Estrés y Procesos Cognitivos. Barcelona: Ediciones Martínez Roca.

Matud, P. (2004). Gender Differences in stress and Coping Styles. Personality and Individual Differences. 37, 1401-1415.

Matthews, G. \& Deary, I. (1998). Stress, Health and medical Psychology. En Matthew y Deary (Eds). Personality Traits. Cambridge: University Press.

Mingote, J., Moreno B. y Gálvez, M. (2004). Desgaste Profesional y Salud de los Profesionales Médicos: Revisión y Propuestas de Prevención. Medicina Clínica, 123 (7). 
Moreno, González y Garrosa (2001). Desgaste Profesional (burnout), personalidad y salud percibida. En J. y R.F. Buendía (Ed). Empleo, Estrés y Salud. Madrid: Psicología Pirámide.

O’Connor, D., O’Connor, R., White, B. \& Bundred, P. (2000). The Effect of Job Strain on british general Practioners' Mental Health. Journal of Mental Health. 9 (6), 637-654.

Pelfrene, E., Vlerick, R., Mak, R., De Smet, Kornitzer, M. \& De Backer, G. (2001). Scale Reliability and Validity of the Karase 'Job Demand-Control-Support' model in the Belstress Study. Work \& Stress, 15 (4), 297-313.

Pérez, C. (1997). Actividades Recreativas y Características Laborales en Pacientes con Infarto al Miocardio. Manuscrito sin publicar, Universidad Simón Bolívar, Caracas, Venezuela.

Roger, D., Jarvis, G. \& Najarian, B. (1993). Detachment and Coping: The construction and validation of a new Scale for Measuring Emotion Control. Personality and Individual Differences. 15 (6). 619-626.

Roger, D. (1995, abril). The mechanics of stress: a model for the relationship between stress, health and personality. Comunicación presentada en la 5ta. Conferencia Internacional en Manejo del Estrés, Noordwijkerhout, Holanda.

Rout, U. (1999). Gender Differences in Stress, Satisfaction and Mental Wellbeing among General Practicioners in England. Psychology, Health \& Medicine, 4 (4), 345-354.

Simpson, L. \& Grant, L. (1991). Sources of Magnitude of Job Stress Among Physicians. Journal of Behavioral Medicine. 14 (1), 27-41.

Smith, T. \& Williams, P. (1992). Personality and Health: Advantages and Limitations of the FiveFactor Model. Journal of Personality. 60, 395-423.

Steptoe, A. (1991a). Psychological coping, individual differences and psychological stress responses. En C.L. cooper and Payne. Personality and Stress: Individual diferences in the stress process. London: Jonh Wiley and Sons.

Steptoe, A. (1991b). The links between stress and illness. Pychosomatic Research, 35, 633-644.

Taylor, S.E. \& Aspinwall, L.G. (1996). Mediating and moderating processes in psychosocial stress: Appraisal, coping, resistance and vulnerability. En H.B. Kaplan (Ed.) Psychosocial stress: Perspectivesonstructure, theory, life-course, andmethods(pp.71-110). SanDiego: AcademicPress.

Yayli, G., Yaman, H. \& Yaman, A. (2003). Stress and Work-Life in a University Hospital in Turkey: Evaluation of the Brief Symptom Inventory and Ways of coping Inventory in Hospital Staff. Social Behaviour and Personality. 31 (1), 91-100. 